\title{
Study of an extended EUV filament using SoHO/SUMER observations of the hydrogen Lyman lines
}

\author{
P. Schwartz ${ }^{1}$, P. Heinzel ${ }^{1}$, B. Schmieder ${ }^{2}$, and U. Anzer ${ }^{3}$ \\ 1 Astronomical Institute, Academy of Sciences of the Czech Republic, 25165 Ondřejov, Czech Republic \\ e-mail: [schwartz; pheinzel] @asu.cas.cz \\ 2 Observatoire de Paris, Section Meudon, LESIA, 92195 Meudon Principal Cedex, France \\ e-mail: brigitte.schmieder@obspm.fr \\ 3 Max-Planck-Institut für Astrophysik, Karl-Schwarzschild-Strasse 1, 85740 Garching, Germany \\ e-mail: ula@mpa-garching.mpg.de
}

Received 16 May 2006 / Accepted 9 August 2006

\begin{abstract}
Context. In previous work we determined the 3D structure of an EUV extension of the filament observed by SoHO/CDS on 15 October 1999. The determination of the 3D structure depends on the optical thickness $\tau_{912}$ at the hydrogen Lyman-continuum edge at $912 \AA$. The $\tau_{912}$ value was unknown, therefore it was taken as a free parameter for the $3 \mathrm{D}$-structure determinations.

Aims. The plasma properties (including $\tau_{912}$ ) of this EUV filament are estimated using non-LTE modelling of profiles of the hydrogen Lyman lines.

Methods. The lines $\mathrm{L} \beta, \mathrm{L} \delta, \mathrm{L} \varepsilon, \mathrm{L} 6$ and $\mathrm{L} 7$ were observed by SoHO/SUMER with the slit positioned across the EUV filament. In our modelling the EUV filament was approximated by a horizontal 1D slab with constant pressure and the temperature decreasing vertically from the edges to interior of the slab. The values of the geometrical thickness of the slab in the EUV extension were taken from its 3D structure determined for the $\tau_{912}$ values estimated iteratively. The synthetic profiles were fitted to observed ones using the $\chi^{2}$ minimization.

Results. For the $\mathrm{H} \alpha$-filament part we obtained the expected results: a small geometrical thickness between several thousand and $15000 \mathrm{~km}$, temperatures around $5000 \mathrm{~K}$ in the interior and around $20000 \mathrm{~K}$ in PCTRs, and pressure $\sim 0.1 \mathrm{dyn}^{-2}$. That leads to an optical thickness in the $\mathrm{H} \alpha$ line center larger than 1. In the EUV extension, temperatures of $10000-40000 \mathrm{~K}$ in the interior and $35000-50000 \mathrm{~K}$ in the vertically extended PCTRs and a pressure of $\sim 0.01 \mathrm{dyn} \mathrm{cm}^{-2}$ were determined. Such large variations of the temperature lead to large variations of $\tau_{912}$.

Conclusions. The EUV extension is inhomogeneous - areas both optically thick and thin at $912 \AA$ are present close to each other. Parts optically thick at $912 \AA$ are visible also in $\mathrm{H} \alpha$ as faint and tiny dark structures around the very dark and well pronounced $\mathrm{H} \alpha$ filament. The volume blocking mechanism is an important mechanism throughout the whole EUV extension even in its optically thick parts. The absorption dominates only in the $\mathrm{H} \alpha$-filament part of the EUV filament.
\end{abstract}

Key words. Sun: filaments - radiative transfer - techniques: spectroscopic - line: profiles

\section{Introduction}

It was found (Heinzel et al. 2001; Schmieder et al. 2003, 2004) that filaments are more extended when observed in EUV spectral lines with wavelengths below the hydrogen Lyman-continuum edge (912 $\AA$ ) than in $\mathrm{H} \alpha$ ( $\mathrm{H} \alpha$ filaments). These extended dark structures observed in EUV lines are called EUV filaments. The intensity depression at the EUV filament can be explained by two mechanisms: absorption and volume blocking.

The first mechanism concerns the absorption in the hydrogen Lyman continuum within the cool filament plasma of the EUV-line radiation emitted from beneath the filament. Therefore only radiation with wavelengths below $912 \AA$ emitted from the transition region (hereafter TR) or corona beneath the filament is absorbed. EUV filaments consist of very dark parts which are cospatial with the $\mathrm{H} \alpha$ filaments and of less dark so-called EUV extensions seen in EUV. The EUV extensions are visible in $\mathrm{H} \alpha$ only faintly or are not visible at all due to low contrast - their optical thickness is small $(<1)$ in this line. Even if they are visible in the $\mathrm{H} \alpha$ they can be observed in this line only as fine structures by instruments with higher spatial resolution (e.g. VTT, SST). However, the optical thickness of the resonance Lyman continuum below $912 \AA$ can produce - for the same column density a clearly observable contrast in the EUV extension. Absorption by the resonance continuum is a process where atoms or ions absorb a photon of energy sufficient for their ionization from the ground level. The energy released by subsequent radiative recombination is redistributed in the whole range of wavelengths of this continuum. This mechanism influences the intensities of EUV lines radiated both from the corona and TR. The contribution of this mechanism to the line intensity depression depends on the properties of the EUV filament (geometrical thickness, filling factor, temperature, plasma density, ionization degree).

The second mechanism - volume blocking - can influence only the intensities of coronal lines. This mechanism is based on the fact that hot coronal line radiation cannot be emitted from the volume of the relatively cool EUV filament. The geometrical thickness of the EUV filament is the basic parameter 
determining the contribution of this mechanism to the line intensity depression. Both the absorption and volume-blocking mechanisms can contribute together to the intensity depression in the EUV filament (Schwartz et al. 2004, hereafter Paper I). A detailed description of these mechanisms can be found in Heinzel et al. (2003), Schmieder et al. (2004) and Anzer \& Heinzel (2003, 2005).

In this paper we continue our investigation (Paper I) of the EUV filament observed on 15 October 1999 by SoHO (Solar and Heliospheric Observatory) in the EUV spectral range and by VTT (Vacuum Tower Telescope) in the $\mathrm{H} \alpha$ line using the MSDP (Multichannel Subtractive Double Pass) spectrograph (Mein 1991, 2002). After reconstruction of the 3D structure of its EUV extension using the SoHO/CDS (Coronal Diagnostic Spectrometer) (Harrison et al. 1995) observations we estimate the properties of the cool plasma using the nonLTE modelling of the hydrogen Lyman lines observed by SoHO/SUMER (Solar Ultraviolet Measurements of Emitted Radiation) (Wilhelm et al. 1995). In our previous work we did not know the Lyman-continuum optical thickness of the EUV filament and therefore we took it as a free parameter in interval from 3 to 7 for the reconstruction of the 3D structure. The ratio of contributions to the line intensity depression of the absorption and volume blocking is also dependent on this optical thickness. In this paper we compute the optical thickness at the edge of the hydrogen Lyman continuum of the EUV filament using the nonLTE modelling of hydrogen Lyman line profiles. The knowledge of this optical thickness makes it possible to improve our general model of the EUV filaments and to find the mechanism that dominates the line intensity depression. In Sect. 2, the observations used in our work are described. Knowledge of the 3D structure of the EUV extension is necessary for the computations of our non-LTE models. Therefore, we briefly explain its calculations in Sect. 3. A short description of the 1D non-LTE model is given in Sect. 4. In Sect. 5, the detailed results of this work are presented and general results are discussed in Sect. 6.

\section{Observations}

\subsection{SoHO/CDS observations}

For the reconstruction of the $3 \mathrm{D}$ structure of the EUV extension, given in Paper I, we used the CDS observations made on 15 October 1999 between 10:01 and 10:52 UT. The SoHO coordinates of the CDS raster center are: solar $X=$ -253.8 arcsec, solar $Y=512.8$ arcsec. The dimensions of the raster are 244 arcsec $\times 240$ arcsec. We call this raster the "large CDS raster" in this paper. Among the observed EUV lines, we chose two coronal lines for our study Mg X $624.94 \AA$ and Si XII $520.60 \AA$ and one TR line O V $629.73 \AA$. A raster of the $\mathrm{Mg} \mathrm{X}$ coronal line is shown in Fig. 1. More details about these CDS observations can be found in Paper I.

The CDS observations in a small raster were then carried out between 11:24-11:55 UT in the same spectral lines; the dimensions of this raster are $122 \operatorname{arcsec} \times 121 \operatorname{arcsec}$. The coordinates of its left lower corner are solar $X=-315 \operatorname{arcsec}$, solar $Y=$ 452 arcsec. The position of the small raster is marked by the dashed-line box in the large raster in Fig. 1. These CDS observations in the He I $584.33 \AA$ line were used for the CDS-SUMER co-alignment.

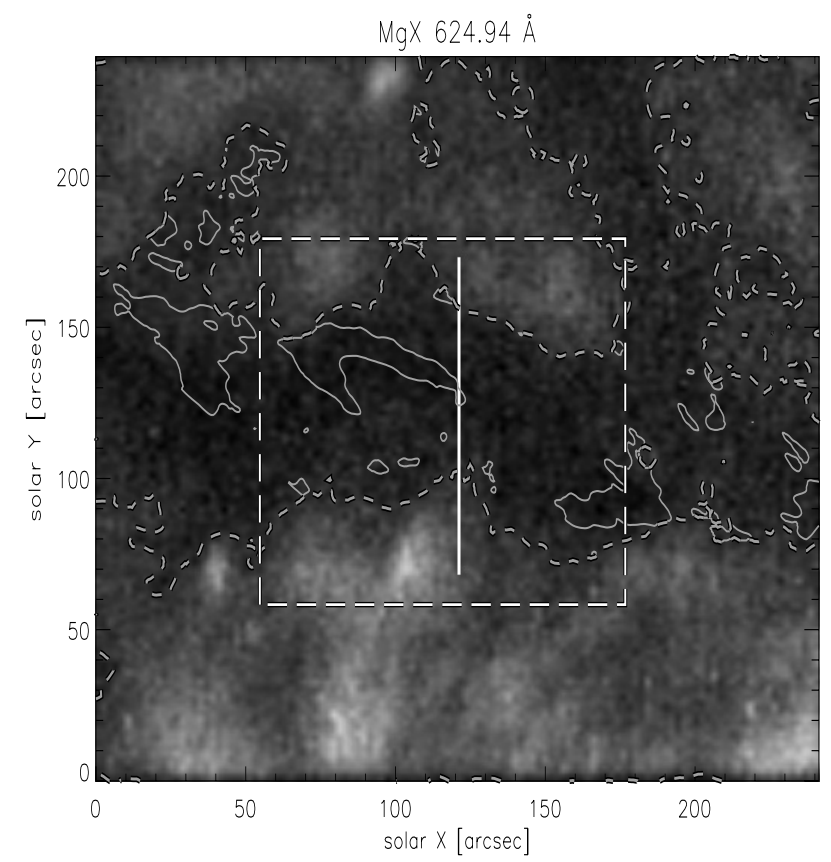

Fig. 1. Large CDS raster of the filament in the EUV coronal line Mg X $624.94 \AA$. The dark EUV filament is clearly seen marked by dashed-line contours. Full-line contours represent the very dark parts of the $\mathrm{H} \alpha$ filament observed by MSDP/VTT (see Fig. 2). The vertical bar shows the position of the SUMER slit during observations of hydrogen Lyman lines. The dashed-line box marks the position of a small CDS raster used for CDS-SUMER co-alignment.

\subsection{SoHO/SUMER observations}

Spectra of hydrogen Lyman lines used for our modelling were detected by SUMER during two sequential observations. Both observations were performed using a slit of $1 \operatorname{arcsec} \times 120 \operatorname{arcsec}$ positioned across the filament; the center of the slit was at solar $X=-245 \operatorname{arcsec}$, solar $Y=515 \operatorname{arcsec}$ and solar $X=$ -243 arcsec, solar $Y=515$ arcsec, respectively. The spectra are shown in Fig. 3. During the first sequence a spectral window at 923-949 A was observed. The observations started at 10:42 UT, lasted $14 \mathrm{~min}$ and 17 exposures were made (each lasted $45 \mathrm{~s}$ ). For our modelling we took spectra of $\mathrm{L} \delta, \mathrm{L} \varepsilon, \mathrm{L} 6$ and $\mathrm{L} 7$ from the last exposure. During the second sequence the spectral window of 1025-1031 $\AA$ was observed. The observations started at 10:56 UT, lasted $1 \mathrm{~min}$ and 2 exposures were made each lasting $30 \mathrm{~s}$. We used spectra of the $\mathrm{L} \beta$ line from the first exposure in order to have them close in time to the observations of other Lyman lines. Both these sequential observations were part of the JOP 017/107 study. During this study the compensation for solar rotation was switched on. In the spectra shown in Fig. 3 several sections are marked. The section $\mathrm{H} \alpha$-fil corresponds to the place where the slit crosses the darkest area of the EUV filament marked by the full-line contour in Fig. 1. This area is clearly visible as a very dark structure also in the $\mathrm{H} \alpha$ line (see Fig. 2). Lyman-line profiles averaged along sections ext1-ext8 were used to model other parts of the EUV filament. Although sections ext7 and ext8 do not belong to the EUV filament according to the $\mathrm{MgX}$ line observations (Fig. 1), they are dark in the TR line $\mathrm{OV}$ as shown in Fig. 4. Therefore the profiles from these two sections were also analyzed. Average profiles from the quiet chromosphere labeled as QS were used to reconstruct the background irradiation of the EUV filament. 


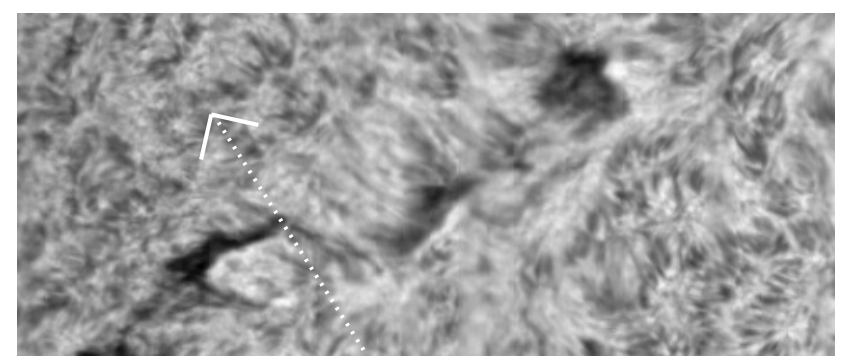

Fig. 2. The filament observed with the VTT/MSDP at 10:16 UT; a map of intensities at the center of the $\mathrm{H} \alpha$ line is shown. Dimensions of the rasters are $366 \operatorname{arcsec} \times 145 \operatorname{arcsec}$ (one pixel is $0.25 \operatorname{arcsec}$ ). The white dotted arrow represents the position of the SUMER slit and also indicates North. The position of the slit is estimated with an error of \pm 10 arcsec in both horizontal and vertical directions. The spatial resolution of this image allows us to resolve fine structures in the vicinity of the well pronounced and very dark $\mathrm{H} \alpha$ filament. The slit crosses many such structures even if taking into account the large inaccuracy of the estimation of the slit position.

For an estimate of the slit position in the CDS rasters a coalignment between SUMER and CDS should be carried out because of the uncertainty in the relative pointing of the two instruments. First, the positions of dark structures in the small CDS raster in the He I $584.33 \AA$ line and in the SUMER raster made in the $\mathrm{L} \delta$ line (hereafter called the $\mathrm{L} \delta$ raster) were compared. We used the small CDS raster rather than the large one because the small CDS raster has similar position, dimensions and pixel sizes as the L $\delta$ rasters. Two L $\delta$ rasters were obtained covering a part of the EUV filament, one before and one after the two sequences of observations described in the previous paragraph. We considered the first one because it is closer in time to the CDS observations of the small raster. Observations of this L $\delta$ raster started at 11:26 UT and lasted $41 \mathrm{~min}$. Its dimensions are $114 \operatorname{arcsec} \times 120$ arcsec and the position of its left lower corner is solar $X=-296$ arcsec, solar $Y=455$ arcsec. Then the estimate of the slit position was improved using the correlation between distributions along the slit of the intensities of two TR lines: O VI 1031.9 $\AA$ observed by SUMER together with $\mathrm{L} \beta$ and OV $629.7 \AA$ observed by CDS. As already shown in Paper I, these two lines correlate well outside the EUV filament only (see Fig. 4) because the O VI line (in difference from the $\mathrm{OV}$ line) is not absorbed by the EUV-filament hydrogen plasma. Finally, the position of the SUMER slit center in the large CDS raster (Fig. 1) was estimated as $X=122 \pm 2$ arcsec and $Y=120 \pm 2$ arcsec.

\section{3D structure of the EUV extension}

The 3D structure of the EUV extension of the filament observed on 15 October 1999 was reconstructed in Paper I using the spectroscopic model of Heinzel et al. (2003). This model is based on the fact that TR and coronal EUV lines with wavelength below the hydrogen Lyman-continuum edge (912 $\AA$ ) are absorbed by relatively cold filament plasma which occupies the volume in which the coronal emission is depressed (blocked). We chose the large ( 244 arcsec $\times 240$ arcsec) CDS rasters in the Mg X $624.94 \AA$ and Si XII $520.60 \AA$ lines for the 3D-structure computations.

The top and bottom heights of the EUV extension above the solar surface result from the spectroscopic model. We calculated both heights in each pixel inside the EUV-extension area of the CDS rasters and thus we could reconstruct the 3D structure of the extension. The smoothed 3D structure of the EUV extension computed for $\tau_{912}=5$ is shown in Fig. 10 in Paper I. The 3D structure obtained directly from heights computed by the spectroscopic model is rather inhomogeneous. It was smoothed in that figure to see a general shape of the EUV extension. The $3 \mathrm{D}$ shape and average heights practically do not change with $\tau_{912}$ over the interval between 1 and 7. For example, one can compare the height maps shown in Fig. 8 in Paper I computed for values 3, 5 and 7 of $\tau_{912}$.

In very dark areas (marked by the full-line contour in Fig. 1) visible clearly in $\mathrm{H} \alpha$ (the optical thickness in the $\mathrm{H} \alpha$ line center being larger than 1.0), the optical thickness $\tau_{912}$ is so large that all radiation emitted from beneath the filament is absorbed. Then only the radiation emitted from the corona above the filament is observed and therefore it is possible to calculate only the height $h_{\mathrm{t}}$ of the top boundary of the $\mathrm{H} \alpha$ filament. The height $h_{\mathrm{t}}$ can be calculated using the formula (Heinzel 2003):

$h_{\mathrm{t}}=-H^{\prime}(\mathrm{Mg} \mathrm{X})\left[\ln I_{\mathrm{fg}}(\mathrm{Mg} \mathrm{X})-\ln I_{\mathrm{qs}}(\mathrm{Mg} \mathrm{X})\right]$,

where $H^{\prime}(\mathrm{MgX})$ is the scale height of intensity decrease of the $\mathrm{Mg}$ X $624.94 \AA$ line in the line-of-sight direction, $I_{\mathrm{fg}}(\mathrm{Mg} \mathrm{X})$ is the integral intensity of radiation at this line emitted from the corona above the $\mathrm{H} \alpha$ filament and $I_{\mathrm{qs}}(\mathrm{Mg} \mathrm{X})$ is the intensity emitted from the corona outside the EUV filament.

\section{Non-LTE radiative transfer used for the modelling}

Our exploratory models use the one-dimensional (1D) radiative transfer, i.e. the filament is approximated by a horizontal $1 \mathrm{D}$ slab (Heinzel et al. 1997). The geometrical scheme of such a model is shown in Fig. 5. The slab is horizontally infinite (homogeneous) and has a finite vertical dimension which is equal to the geometrical thickness of the filament. $h_{\mathrm{b}}$ and $h_{\mathrm{t}}$ are heights above the solar surface of the bottom and top boundaries of the 1D slab, respectively. In Heinzel et al. (2003) and in Paper I, these heights were denoted as $h_{1}$ and $h_{2}$ at the $\mathrm{H} \alpha$ filament and as $h_{3}$ and $h_{4}$ at the EUV extension. We assume the following properties of the filament slab model:

- the slab is irradiated only from the solar atmosphere beneath, i.e. no coronal radiation plays a role in our computations;

- there are two identical prominence-corona transition regions (PCTR), one on the top, another at the bottom of the slab;

- the temperature decreases from both surfaces to the slab interior symmetrically;

- the temperature gradient is steep in PCTRs, in the slab interior the temperature distribution is rather flat;

- the pressure is supposed to be constant in the whole slab (isobaric models);

- the micro-turbulent velocity is not larger than $10 \mathrm{~km} \mathrm{~s}^{-1}$ in the whole slab.

The temperature variation with height $h$ across the slab is expressed by an empirical formula:

$T(h)=T_{\mathrm{c}}+\left(T_{\mathrm{s}}-T_{\mathrm{c}}\right)\left[1-4 \frac{h}{D}\left(1-\frac{h}{D}\right)\right]^{\gamma}$,

where $T_{\mathrm{s}}$ is the temperature at the surfaces of the slab, $T_{\mathrm{c}}$ the temperature in the slab interior, $D$ the geometrical thickness and $\gamma$ determines the temperature gradient. Larger $\gamma$ means a steeper temperature rise and thinner PCTR.

To solve the equation of radiative transfer and equations of statistical equilibrium we used the MALI (Multilevel 


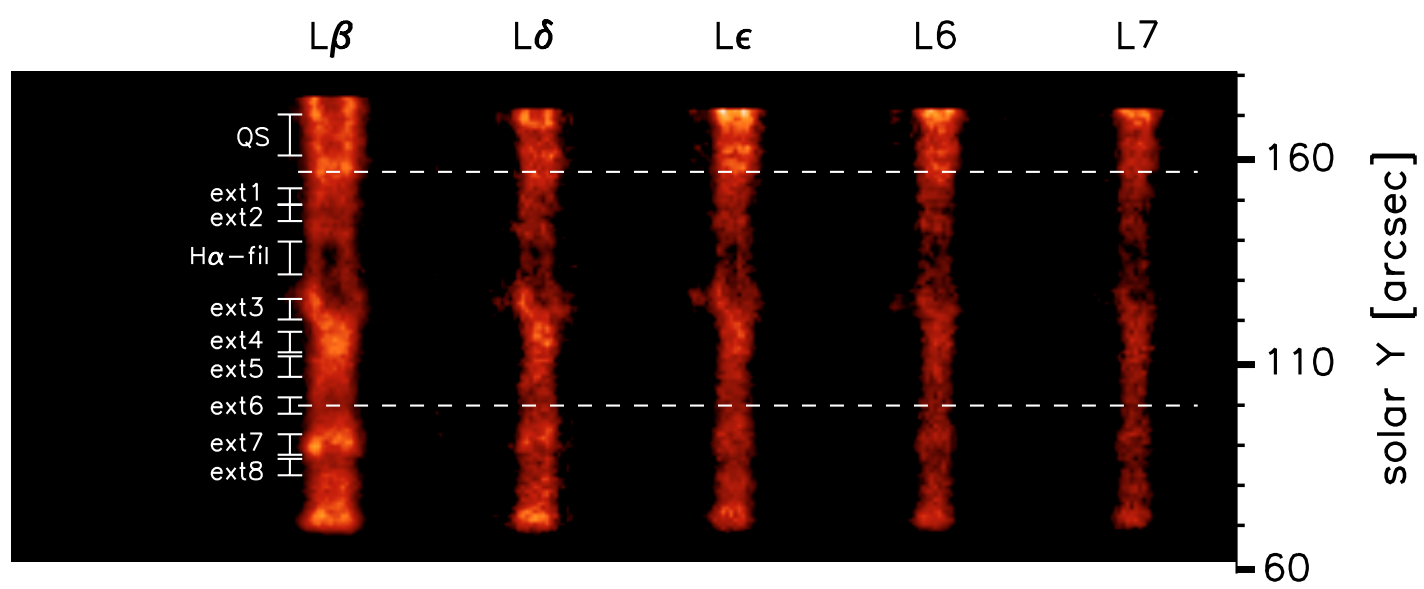

Fig. 3. SUMER spectra of hydrogen Lyman lines. The slit was positioned across the EUV filament bordered by two horizontal dashed lines in the spectra. These lines correspond to borders of EUV-filament area fixed in the Mg X raster shown in Fig. 1 (bordered by the dashed-line contour). The position on the slit is expressed by the coordinate solar $Y$ of that CDS raster. For other details see the text.

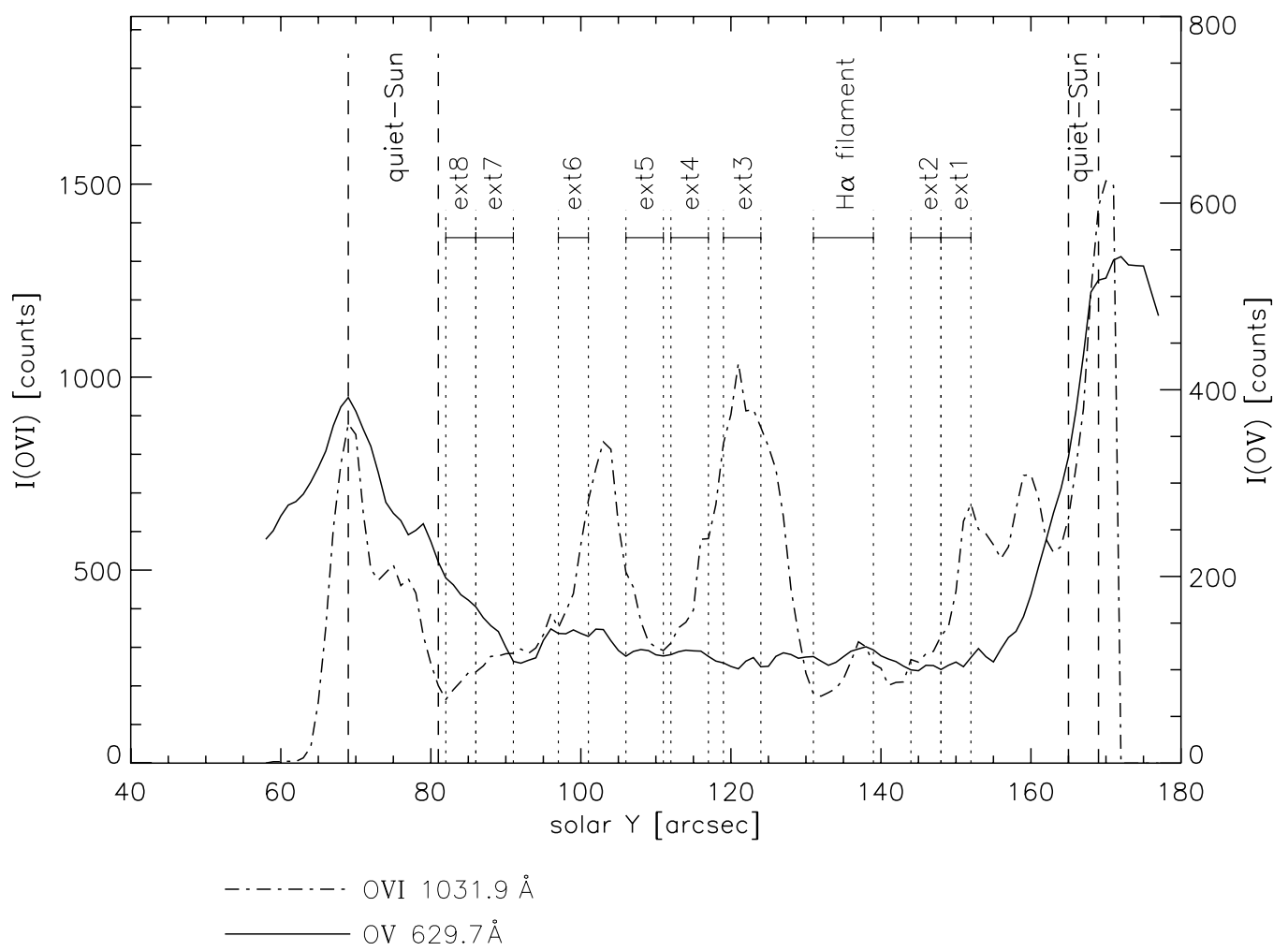

Fig. 4. Comparison of intensity distributions of the O VI $1031.9 \AA$ and O v $629.7 \AA$ spectral lines along the SUMER slit positioned as shown in Fig. 1. On the abscissa are the positions along the SUMER slit in coordinates of the CDS raster. Two quiet-Sun sections are marked by the vertical dashed lines. Between these two sections the slit crosses the EUV filament. All EUV-filament sections (H $\alpha$-fil, ext1-ext8) are marked and all of them are in the area between the two quiet-Sun sections. The intensities are in counts because the influence of noise can be estimated from the counts using the Poisson statistics. The intensities of the $\mathrm{O}$ VI and $\mathrm{O} v$ lines in erg $\mathrm{cm}^{-2} \mathrm{~s}^{-1} \mathrm{sr}^{-1}$ can be calculated by multiplying counts by factors of 0.3 and 3.7, respectively. Because of higher counts the OVI line is less influenced by noise than the OV line although intensities in physical units of the $\mathrm{O}$ VI line are lower by a factor 5 than the intensities of the $\mathrm{O} v$ line. The distributions of both lines were scaled into one plot to show their good correlation in the quiet-Sun regions.

Accelerated Lambda Iterations) method (Rybicki \& Hummer 1991; Heinzel 1995; Paletou 1995) with a model of the hydrogen atom consisting of 12 levels plus continuum. The slab was divided into 87 horizontal layers in order to optimize the optical depths in the $\mathrm{L} \alpha$ line center. The solution of the equation of radiative transfer was performed in two steps, using the same frequencies as for the background irradiation.
First, the source functions were determined using the Feautrier method for radiative transfer and the MALI technique based on preconditioning (the ionization is computed according to Heinzel 1995). For the $\mathrm{L} \alpha$ and $\mathrm{L} \beta$ lines the partial frequency redistribution was used (Paletou 1995), while higher Lyman lines were treated with a complete redistribution. Average disk profiles of the hydrogen Lyman lines of Warren et al. (1998) were used for background irradiation of the 


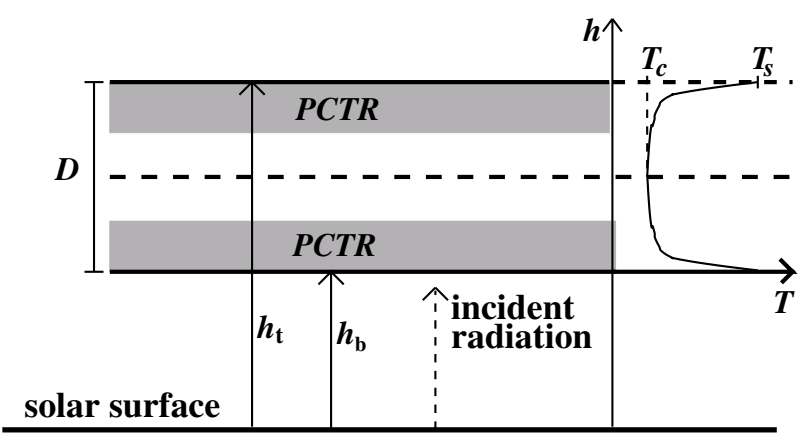

Fig. 5. Scheme of the 1D-slab model used for the computation of the profiles of hydrogen Lyman lines. A description of this scheme and the model itself can be found in the text

slab, but for $\mathrm{L} \alpha$ we still use the OSO- 8 profile as in Heinzel (1995).

In the second step the formal solution along the line of sight was performed. The line profiles were computed at the same frequencies as used for the background radiation. In order to facilitate the search for the best fit, the same frequencies are also used for the observed line profiles.

\section{Results}

\subsection{Reconstruction of the hydrogen Lyman line profiles of background irradiation}

For the second step of our solution of the non-LTE radiative transfer it is necessary to know the background irradiation of an EUV filament. But it is possible to see through the EUV filament only at the far wings of the hydrogen Lyman lines. In the central and slope parts of the profiles the EUV filament is more or less optically thick. So an often used way of estimation of background radiation is to take average profiles from quiet areas in close vicinity of the EUV filament. But when we compared average profiles from the quiet-chromosphere QS section at the SUMER slit with profiles from the EUV-filament sections ext1-ext8 and $\mathrm{H} \alpha$-fil we found that there is a rather large difference also in far wings where the EUV filament is optically thin. An example of such a discrepancy is shown in Fig. 6 for the $\mathrm{L} \delta$ line of the ext2 section. There we plot (with a dotted line) the synthetic profile computed using the 1D-slab model without any background irradiation in the second step of computations. This is the profile of radiation emitted just from the EUV filament itself. Then the EUV filament is optically thin at wavelengths where the intensity of this profile reaches zero. In the example shown in Fig. 6 the EUV extension is optically thin for the $L \delta$ line for $|\Delta \lambda|>0.2 \AA$ around the line center. But the average profile for the quiet chromosphere differs remarkably at these wavelengths from the profile averaged along the section ext2. The same problem occurs when comparing the quiet-chromosphere profiles with profiles averaged along any of the EUV-filament sections for all hydrogen Lyman lines we used. Therefore the quiet-chromosphere profiles were not acceptable for use as the background irradiation of the EUV filament. Therefore the profiles of the background irradiation had to be reconstructed.

The reconstruction of the profiles of the background irradiation was made by adjusting the wings and central parts of the quiet-chromosphere profiles using different factors. At the optically thin wings the profiles of the background irradiation should be equal to the profiles of the EUV extension. Therefore we

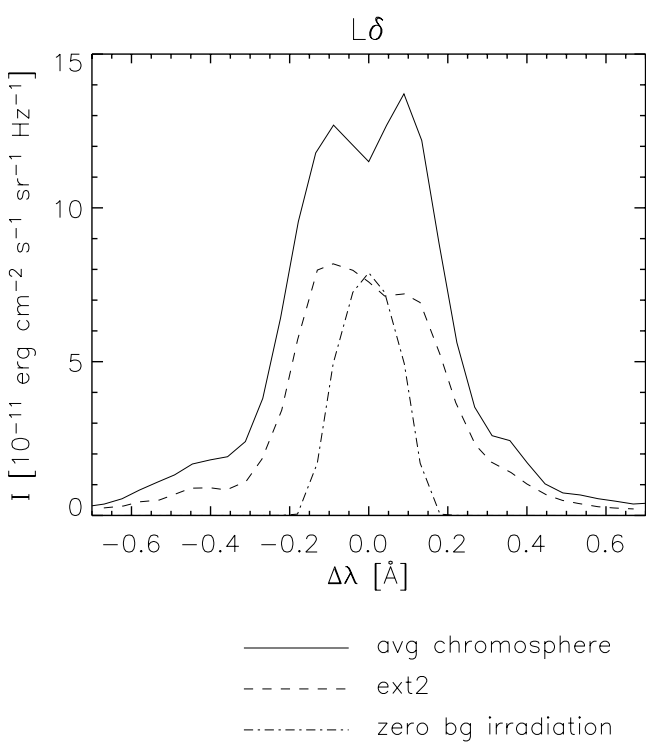

Fig. 6. Comparison of $L \delta$ profiles from quiet chromosphere and from the EUV filament. The profile labeled "avg chromosphere" is the average profile from the quiet-chromosphere section at the SUMER slit named QS (see Fig. 3). Profile labeled "ext2" is the profile from the section ext2. The synthetic profile computed using our 1D-slab model in the case of no background irradiation is labeled "zero bg irradiation"; a filament is optically thin at wavelengths where this profile reaches zero.

multiplied the values of intensity in the wings by factors such that the average intensities there were the same as in the EUV-filament profiles. For each reconstructed profile, different multiplicative factors for right and left wings were estimated. The optically thick central parts of the profiles were reconstructed by comparison with the profiles of Warren et al. (1998) observed in different quiet-Sun regions. These authors give 6 profiles with different intensities in the wings and center for each hydrogen Lyman line from $\mathrm{L} \beta$ to $\mathrm{L} 11$. The procedure of reconstruction the optically thick central part of the profile of the background irradiation for any Lyman line was as follows: Using the 6 quiet-Sun profiles of Warren et al. (1998) for this line we plotted 6 points of average intensity of the optically thick central part on the intensity at some chosen wavelengths in both wings. We constructed such plots for several (3-4) wavelengths chosen in the optically thin wings. Then in each plot we connected those 6 points using linear interpolation and obtained the dependences of average intensities of the optically thick central part on the intensities in the wings. We call these dependences the "wing-center intensity plots". For example, the "wing-center intensity plot" for the L $\delta$ line is shown in Fig. 7. Then for the intensities of the EUV-filament profile in each chosen wavelength in the wings we found the corresponding average intensities of the central optically thick part using the "wing-center intensity plots". As a final average intensity of the central optically thick part we took an average of all average intensities of the optically thick central part estimated for all chosen wavelengths in the wings. Finally we multiplied the intensities in the central part of the quiet-chromosphere profile by such a constant that the average intensity in the central part of the reconstructed profile was equal to the final average intensity of the optically thick central part. An example of the reconstructed $\mathrm{L} \delta$ line profile of the background radiation for the section ext2 is shown in Fig. 8. For comparison the average filament $\mathrm{L} \delta$ profile from the same section is plotted in this figure. 


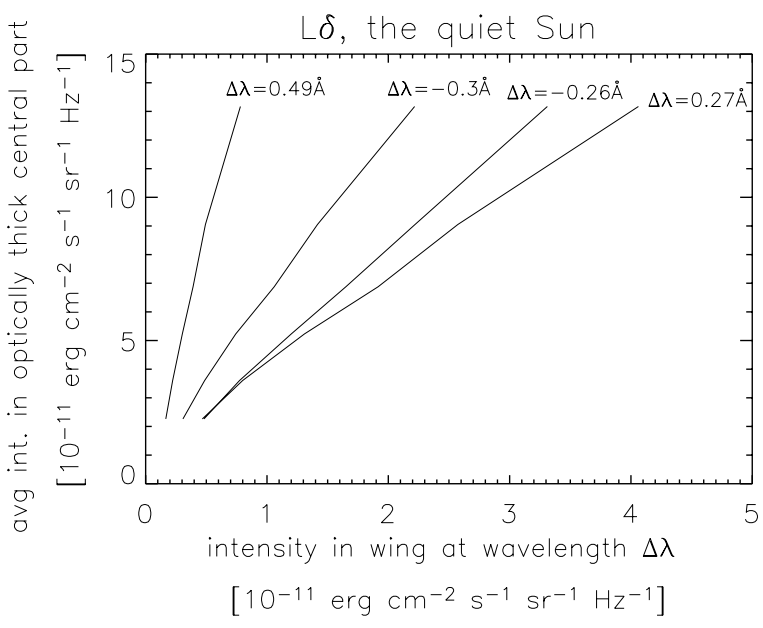

Fig. 7. The "wing-center intensity plot" for the $L \delta$ line. This plot shows dependence of the average intensity in the optically thick central part of line (ordinate) on the intensity at chosen wavelengths $\Delta \lambda$ in line wings (abscissa). This "wing-center intensity plot" was created using the quiet-Sun L $\delta$ asymmetric profiles of Warren et al. (1998).

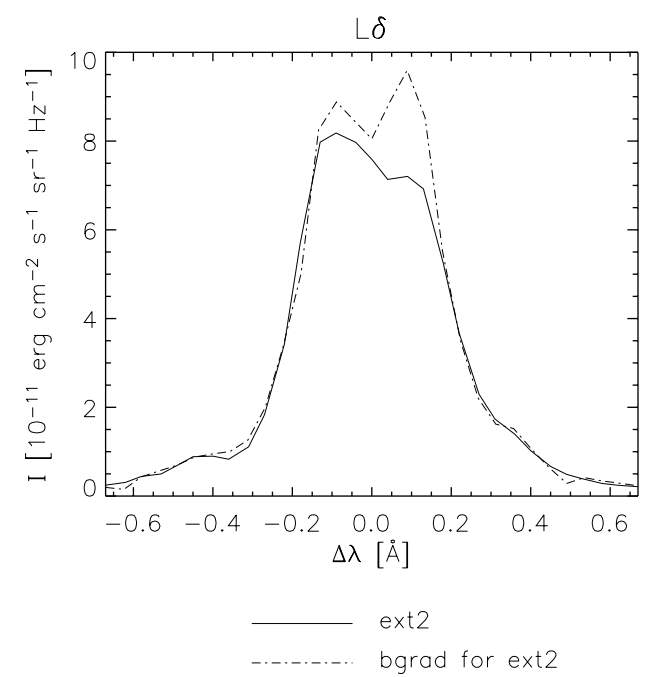

Fig. 8. Comparison of the L $\delta$ profile from the EUV filament with the reconstructed profile for background irradiation. The profile labeled "ext2" is the profile from the section ext2. The reconstructed profile for background irradiation is labeled "bgrad for ext2".

\subsection{Fitting the observed hydrogen Lyman line profiles with synthetic ones computed with the 1D-slab model}

We computed grids of the non-LTE 1D-slab models (described in Sect. 4) for all EUV-filament sections (ext1-ext8 and $\mathrm{H} \alpha$-fil) along the SUMER slit, for different sets of input parameters. The input parameters of the models are:

- $h_{\mathrm{b}}$ - height of the bottom border of the slab above the solar surface (in km);

- $D$ - geometrical thickness of the slab (in $\mathrm{km}$ );

- $T_{\mathrm{s}}, T_{\mathrm{c}}$-temperatures at the border (at the edge between PCTR and corona) and in the interior of the slab;

- $\gamma$-temperature-gradient factor (see Eq. (2));

- $p$-gas pressure (uniform for our isobaric models);

- filling factor - a fraction of the geometrical thickness of the slab filled with cool EUV-filament plasma (not the whole volume of the filament is occupied by cool plasma). There can exist cavities with very low particle density or pores filled with hotter plasma. The presence of such inhomogeneities reduces the total geometrical thickness of the cool plasma responsible for absorption and this reduces the contribution of absorption to the intensity depression. Therefore $D$ derived from the 3D structure is multiplied by a filling factor in the interval $0-1$;

- $v_{\mathrm{t}}$ - turbulent velocity. We assume that it is not higher than $10 \mathrm{~km} \mathrm{~s}^{-1}$. The hydrogen Lyman line profiles are almost insensitive to such low values of $v_{\mathrm{t}}$. Therefore we considered an average value of $5 \mathrm{~km} \mathrm{~s}^{-1}$ for all models

For the profiles of the background radiation used in the second step of the model computations we took the profiles reconstructed from profiles of the QS region as described in Sect. 5.1.

Grids of models were computed using the input parameters in a sufficiently large parameter space. Then we fitted the observed profiles by searching in these grids for a model with the smallest $\chi^{2}$ defined as

$\chi^{2}=\sum_{l=1}^{n_{\mathrm{L}}} \sum_{i=1}^{n_{\mathrm{w}}(l)} \frac{\left[I_{l}^{\mathrm{obs}}\left(\lambda_{i}\right)-I_{l}^{\mathrm{synt}}\left(\lambda_{i}\right)\right]^{2}}{\left[\delta I_{l}^{\mathrm{obs}}\left(\lambda_{i}\right)\right]^{2}}$,

where $I_{l}^{\mathrm{obs}}\left(\lambda_{i}\right)$ and $I_{l}^{\mathrm{synt}}\left(\lambda_{i}\right)$ are the intensities of observed and computed profiles of the line $l$ at wavelength positions $\lambda_{i}$. $\delta I_{l}^{\text {obs }}\left(\lambda_{i}\right)$ is the uncertainty of measurements of the observed line intensity. $n_{\mathrm{L}}$ is the number of the observed spectral lines (in this work we use 5 Lyman lines) and $n_{\mathrm{w}}(l)$ is the number of wavelength positions in which the profile of the line $l$ was observed.

We do not take into account any macroscopic velocities (motions of the whole filament or parts) because it would increase the number of input parameters very much. The macroscopic velocity causes a shift and an asymmetry of the line profiles. The fitting method - searching for the model with the minimal $\chi^{2}-$ reduces any influences of the velocities. Therefore we could neglect the influence of macroscopic velocities on the modelled profiles. We performed a convolution of each synthetic profile from the grids of models with the instrumental profile. These synthetic profiles were then used for $\chi^{2}$ computations (Eq. (3)). We assume that the instrumental profile is mainly dependent on the width of the slit and its dependences on settings of the optical system of the spectrograph are negligible. Therefore we took as an instrumental profile the normalized box symmetrically placed around the center of the spectral line. The width of this box is equal to the width of the slit projected onto the image of the spectra at the detector plane. During the observations of all 5 hydrogen Lyman lines, the slit of width 1 arcsec was used. In the spectral range of interest the angular scale is around $1.03 \mathrm{arcsec} / \mathrm{pixel}$ and the spectral scale is around $45 \mathrm{~m} \AA$ per pixel (Wilhelm et al. 1995). Then the width of the slit projected onto the image of the spectra at the detector plane is equal to $0.04 \AA$. All observed hydrogen Lyman lines have widths of profiles much larger (around 10 times) than the width of the instrumental profile. Therefore the widths of the whole profiles are practically not influenced. But narrow peaks in profiles with reversals can be broadened. The results of fitting of hydrogen Lyman lines using the slab model for all EUV-filament sections are listed in Table 1.

Using the input parameters of the model that computed the synthetic profiles fitting the observed ones, it was possible also to calculate the distributions of plasma properties, such as temperature, hydrogen plasma density, density of particles, density of free electrons and ionization degree of hydrogen, across the slab. For the computations of particle density the hydrogen and helium atoms, protons and electrons were taken into account. The plasma properties were computed with the following assumptions: the ratio $N(\mathrm{He}) / N(\mathrm{H})$ is taken as 0.1 and the plasma of 
Table 1. Results of the fitting of hydrogen Lyman line profiles using the 1D-slab model for all EUV-filament sections ext1-ext8 and H $\alpha$-fil.

\begin{tabular}{c|ccccccccc}
\hline \hline $\begin{array}{c}\text { Section on } \\
\text { the SUMER slit }\end{array}$ & $\begin{array}{c}h_{\mathrm{b}} \\
{[\mathrm{km}]}\end{array}$ & $\begin{array}{c}D \\
{[\mathrm{~km}]}\end{array}$ & $\begin{array}{c}T_{\mathrm{c}} \\
{[\mathrm{K}]}\end{array}$ & $\begin{array}{c}T_{\mathrm{s}} \\
{[\mathrm{K}]}\end{array}$ & $\gamma$ & $\begin{array}{c}P \\
{\left[\mathrm{dyn} \mathrm{cm}^{-2}\right]}\end{array}$ & Filling factor & $\tau_{\mathrm{o}}(\mathrm{H} \alpha)$ & $\tau_{912}$ \\
\hline ext1 & 5300 & 38500 & 10000 & 50000 & 4.0 & 0.015 & 0.7 & 0.499 & 5.52 \\
ext2 & 5400 & 38500 & 9000 & 45000 & 3.0 & 0.015 & 0.4 & 0.306 & 4.55 \\
H $\alpha$-fil & $\cdots$ & $3500^{*}$ & 4000 & 17000 & 6.5 & 0.08 & $\cdots$ & 5.43 & 274 \\
ext3 & $\cdots$ & $15000^{*}$ & 6000 & 22000 & 25.0 & 0.06 & $\ldots$ & 7.45 & 448 \\
ext4 & 9300 & 26500 & 40000 & 44000 & 0.8 & 0.012 & 0.8 & $2.87 \mathrm{E}-4$ & $1.1 \mathrm{E}-2$ \\
ext5 & 25200 & 14300 & 10000 & 35000 & 2.0 & 0.02 & 0.6 & 0.251 & 2.56 \\
ext6 & 11400 & 37700 & 30000 & 45000 & 1.0 & 0.007 & 0.7 & $8.54 \mathrm{E}-4$ & $2.75 \mathrm{E}-2$ \\
ext7 & 14200 & 41300 & 10000 & 45000 & 0.3 & 0.02 & 0.08 & $1.09 \mathrm{E}-2$ & 0.157 \\
ext8 & 17500 & 43000 & 8000 & 50000 & 0.3 & 0.009 & 0.5 & $2.64 \mathrm{E}-2$ & 0.397 \\
\hline
\end{tabular}

* The total geometrical thickness $D_{\text {abs }}$ of the slab layers filled only with a cool filament plasma - it is equal to $D$ multiplied by the filling factor.

the EUV filament is supposed to be in charge equilibrium - the number of protons is the same as the number of free electrons.

The observed profiles of the lines $\mathrm{L} \beta-\mathrm{L} \varepsilon$ from the sections $\mathrm{H} \alpha$-fil (left column in Fig. 9) and ext3 show very deep reversals. Therefore we assumed that both belong to the $\mathrm{H} \alpha$ filament. For $\mathrm{H} \alpha$ filaments the optical thickness in the center of the $\mathrm{H} \alpha$ line is assumed to be larger then 1 therefore it is not possible to compute the geometrical thickness $D$ using the spectroscopic model (Heinzel et al. 2003). Only values of the height $h_{\mathrm{t}}$ of the top boundary of the filament were calculated at these sections using Eq. (1). For these calculations it is necessary to know the scale height of the intensity decrease of the $\mathrm{MgX}$ line and intensities $I_{\mathrm{fg}}(\mathrm{Mg} \mathrm{X})$ and $I_{\mathrm{qs}}(\mathrm{Mg} \mathrm{X})$ measured at the $\mathrm{H} \alpha$ filament and at the vicinity of the EUV filament, respectively. The scale height $H(\mathrm{MgX})$ in the radial direction was estimated from observations published by Fludra et al. (1999). We transformed the scale height $H(\mathrm{Mg} \mathrm{X})$ into the line-of-sight direction (see Paper I) and obtained the value $H^{\prime}(\mathrm{Mg} \mathrm{X})=41100 \mathrm{~km}$. For the quantity $I_{\mathrm{fg}}(\mathrm{Mg} \mathrm{X})$ we used values of 23.8 and $23.3 \mathrm{erg} \mathrm{cm}^{-2} \mathrm{~s}^{-1} \mathrm{sr}^{-1}$ averaged along the sections $\mathrm{H} \alpha$-fil and ext 3 , respectively. For the quantity $I_{\mathrm{qs}}(\mathrm{Mg} \mathrm{X})$ the value $75 \mathrm{erg} \mathrm{cm} \mathrm{cm}^{-2} \mathrm{~s}^{-1} \mathrm{sr}^{-1}$ was estimated as an average intensity from the quiet-Sun regions in the vicinity of the EUV filament (Paper I). Then using Eq. (1) we computed the height $h_{\mathrm{t}}$ of $45700 \mathrm{~km}$ and $48000 \mathrm{~km}$ in the sections $\mathrm{H} \alpha$-fil and ext 3 , respectively. From Eq. (1) it follows that the height $h_{\mathrm{t}}$ is independent of absorption therefore we do not need any initial estimates of $\tau_{912}$ to start our non-LTE modelling. In the constructions of the grids of models we took the geometrical thickness as a free input parameter of the slab model. Instead of the geometrical thickness $D$ of the whole slab it was possible to estimate the total geometrical thickness $D_{\text {abs }}$ of slab layers filled with the cool filament plasma only $-D_{\text {abs }}$ is equal to $D$ multiplied by the filling factor. The hydrogen Lyman lines are emitted almost entirely from beneath the filament and therefore they are influenced only by absorption - not by volume blocking. Therefore they are sensitive to $D_{\text {abs }}$ instead of $D$. We tested the possibility of solving the problem of estimation of $D$ by taking the bottom height $h_{\mathrm{b}}$ of the slab as another free input parameter for the construction of grids of models. But this height determines only the dilution factor for the irradiation field of the slab. Therefore the model is much less sensitive to this height than to $D_{\text {abs }}$. Therefore the obtained values of $h_{\mathrm{b}}$ would be rather uncertain. The results of our modelling of the $\mathrm{H} \alpha$ filament are similar to the results of Schmieder et al. (2003) who describe another filament observed in the $\mathrm{H} \alpha$ line and in EUV lines on 5 May 2000. Also we computed a small geometrical thickness (of several thousands $\mathrm{km}$ ) in the darkest part of the $\mathrm{H} \alpha$ filament, with the optical thickness at the $\mathrm{H} \alpha$ line center above unity, relatively high pressure $\left(\sim 0.1 \mathrm{dyn} \mathrm{cm}^{-2}\right)$, low temperatures (around $10000 \mathrm{~K}$ ) and geometrically thin PCTRs (both PCTRs occupied only 10-30\% of the geometrical thickness of the filament). Only the temperature $T_{\mathrm{c}}=4000-6000 \mathrm{~K}$ estimated for the $\mathrm{H} \alpha$-filament interior in our work is rather low. The value of $8000 \mathrm{~K}$ adopted by Schmieder et al. (2003) seems to be more reliable. Our underestimate of this temperature can be explained by the fact that if there is a hot (relative to the filament interior) PCTR on the top of the filament, its influence on the Lyman line profiles is dominant. In such a case the model is less sensitive to the values of the interior temperature lower then $10000 \mathrm{~K}$, if there is a temperature around $20000 \mathrm{~K}$ in the PCTR. For a better estimation of the temperature in the interior of the $\mathrm{H} \alpha$ filament it would be necessary also to have a profile of the $\mathrm{H} \alpha$ line (as in Schmieder et al. 2003) which is sensitive mainly to low temperatures. Therefore, our modelling is more sensitive to the PCTR. The value of $\tau_{\mathrm{o}}(\mathrm{H} \alpha)$ is larger than 1 and therefore this part of the EUV filament is visible in $\mathrm{H} \alpha$ as the well pronounced $\mathrm{H} \alpha$ filament. Also the ratio of $\tau_{912}$ to $\tau_{\mathrm{o}}(\mathrm{H} \alpha)$ is $50-60$ which is consistent with the results of Schmieder et al. (2003), but it is considerably larger than the values calculated by Anzer \& Heinzel (2005). This result is probably due to the extremely low temperatures that we obtained for these structures visible in $\mathrm{H} \alpha$. An example of fitting the profiles observed in the $\mathrm{H} \alpha$ filament is shown in the left column of Fig. 9. Plots of distributions of plasma properties across the slab for the section $\mathrm{H} \alpha$-fil are shown in the left column of Fig. 10. For the section ext 3 the line profiles with deep reversals and distributions of plasma properties across the 1D slab with geometrically thin PCTRs are similar to those of the $\mathrm{H} \alpha$-fil section.

Values of the geometrical thickness $D$ and height $h_{\mathrm{b}}$ for sections ext 1 , ext 2 and ext4-ext8 were derived from the 3D structure (Sect. 3) of the EUV extension calculated using the spectroscopic model. We did not know the values of the optical thickness $\tau_{912}$ necessary for height and geometrical thickness computations. Therefore we used a so-called average 3D structure $-D$ and $h_{\mathrm{b}}$ were averaged by the values computed for $\tau_{912}$ from 0.1 to 3 . The upper limit of this interval is a threshold of visibility of the EUV filament in the $\mathrm{H} \alpha-\tau_{912}=3$ roughly corresponding to the optical thickness in the $\mathrm{H} \alpha$ line center equal to 0.1 (Anzer \& Heinzel 2005). Our non-LTE model also provides values of the optical thickness of the 1D slab at the centers of the hydrogen lines and at the edge of the hydrogen Lyman continuum. The value of optical thickness $\tau_{\mathrm{o}}(\mathrm{H} \alpha)$ at the $\mathrm{H} \alpha$ line center is useful for distinguishing whether the filament is visible in $\mathrm{H} \alpha$ (for $\tau_{\mathrm{o}}(\mathrm{H} \alpha)$ larger than 0.1 ). The value of $\tau_{912}$ as the output of the non-LTE model can be used for computations 

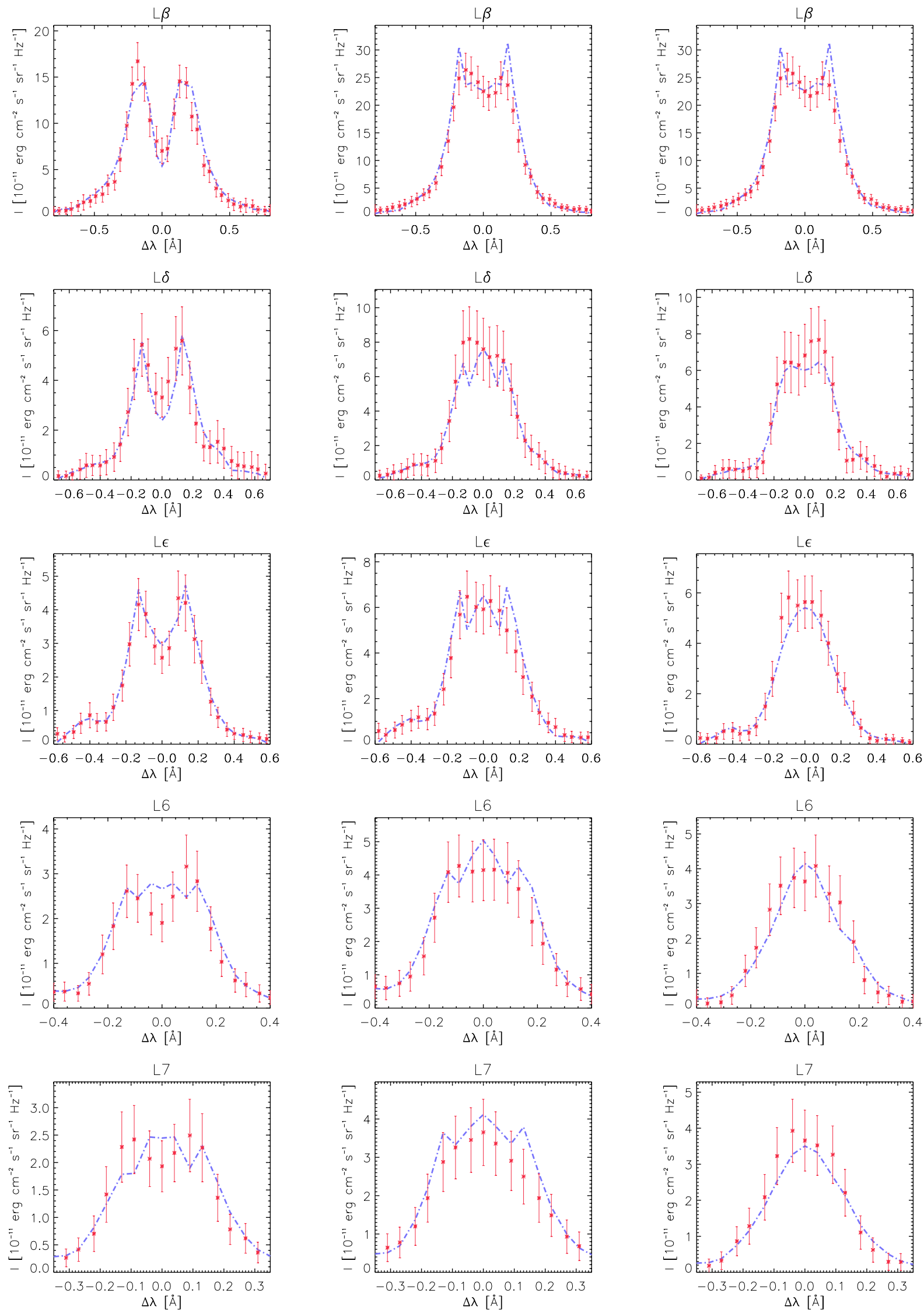

Fig. 9. Comparison of observed profiles from the sections $\mathrm{H} \alpha$-fil, ext2 and ext6 (columns from left to right) with fitted synthetic profiles computed using the 1D-slab model. Observed intensities are plotted with points with errorbars and synthetic profiles with dot-dashed lines. Intensities are expressed in units $10^{-11} \mathrm{erg} \mathrm{cm}^{-2} \mathrm{~s}^{-1} \mathrm{sr}^{-1} \mathrm{~Hz}^{-1}$. 
of the new values of $D$ and $h_{\mathrm{b}}$ using the spectroscopic model (Heinzel et al. 2003). If these new values differ very much from those used as input into the non-LTE model, the profiles are fitted once again using a new grid of models computed with the new values of $D$ and $h_{\mathrm{b}}$. Once again the value of the $\tau_{912} \mathrm{ob}-$ tained from the new fitting is used for computations of $D$ and $h_{\mathrm{b}}$. If there are changes of the $D$ and $h_{\mathrm{b}}$ values the profiles are fitted again, etc. The iterations stop when changes of the $D$ and $h_{\mathrm{b}}$ values between steps are smaller than prescribed values; we stopped the iterations when changes of both values of $D$ and $h_{\mathrm{b}}$ did not exceed $15 \%$.

In the sections ext1, ext 2 and ext5 the EUV extension is optically thick at the hydrogen Lyman-continuum edge. In the sections ext 1 and ext 2 the bottom boundary of the EUV extension is located rather low - at height $h_{\mathrm{b}}$ around $5000 \mathrm{~km}$ above the solar surface. But the EUV extension is vertically wide there and the geometrical thickness $D$ is around $38000 \mathrm{~km}$. Temperatures range from $10000 \mathrm{~K}$ in the slab interior to $50000 \mathrm{~K}$ in PCTRs and the gas pressure is around $0.015 \mathrm{dyn} \mathrm{cm}^{-2}$. In the section ext5 the EUV extension is placed much higher and $D$ is 2.7 times smaller. But the temperature in the PCTRs is lower $(35000 \mathrm{~K})$ and the gas pressure 1.3 times larger than in ext1 and ext 2 - such properties lead to an approximately 2 times larger plasma density. For these three sections the ionization degree of hydrogen was around 0.9 in the interior of the EUV extension. The $\gamma$ parameters are 2-4 which implies geometrically thick PCTRs (both PCTRs occupy 40-60\% of the geometrical thickness of the slab). But there is still a geometrically thick interior of the filament containing $10 \%$ neutral hydrogen. It is interesting to compare the contribution of the absorption and volume blocking to the depression of intensities of EUV coronal lines. We computed the relative contribution of the absorption to the intensity depression of the Mg X $624.94 \AA$ line using formulae derived from the spectroscopic model in Paper I. In the sections ext 1 and ext 2 the contribution of the absorption is only around $16 \%$ and in the ext5 it is around $50 \%$. From this it follows that although the EUV extension is optically thick at $912 \AA$, the volume blocking always plays a crucial role or even dominates over absorption. The reason is that the EUV extension is vertically extended as opposed to the geometrically thin $\mathrm{H} \alpha$ filament where absorption dominates. For the $\mathrm{OV}$ and $\mathrm{Mg} \mathrm{X}$ lines with wavelengths close to each other, the intensity depressions caused by absorption are almost the same. It follows from our modelling that the entire EUV extension is above TR. Therefore the volume blocking cannot affect TR lines and the absorption alone causes only a small intensity depressions of the OV TR line as it is seen in Fig. 4. Even for wavelengths closer to $912 \AA$ the contribution of absorption would be only 1.5 times larger. And for EUV spectral lines with wavelengths lower than the wavelength of the Mg X line (e.g. Si XI $520.60 \AA$ ) the absorption would be even smaller. The profiles from section ext 2 fitted with synthetic profiles are shown in the middle column of Fig. 9. Plots of distributions of plasma properties for this section are shown in the middle column of Fig. 10. For sections ext1 and ext5, the hydrogen Lyman line profiles with shallow reversals and the distributions of plasma properties with geometrically thick PCTRs are similar to those of section ext2.

In sections ext4, ext6, ext7 and ext8 the EUV extension is optically thin at the hydrogen Lyman-continuum edge. In sections ext4 and ext6 the EUV extension occupies a large volume with plasma of high temperature $(\sim 40000 \mathrm{~K})$ and low gas pressure $\left(\sim 0.01 \mathrm{dyn} \mathrm{cm}^{-2}\right)$ and almost the all hydrogen is ionized. In sections ext7 and ext8, temperatures are similar to those of the sections ext1, ext2 and ext5, the gas pressure varies

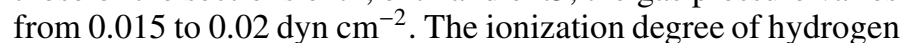
is around 0.9 in the filament interior but it is close to 1 in the PCTRs. The parameter $\gamma$ is small $(\leq 1)$ therefore the PCTRs are geometrically very thick for sections ext4, ext6, ext7 and ext8. In such cases there is only a very small amount of neutral hydrogen able to absorb. Therefore the EUV extension is optically thin at the Lyman continuum edge in these sections. But both sections ext 4 and ext6 belong to the dark structure of the EUV filament observed in the $\mathrm{MgX}$ line by CDS (Fig. 1). Therefore the intensity depression can be caused only by volume blocking in these sections. Volume blocking can be very effective in reducing the coronal line intensity because the EUV extension is vertically very large in sections ext4, ext6, ext7 and ext8 - the geometrical thickness ranges from 26000 to $43000 \mathrm{~km}$. The profiles of the hydrogen Lyman lines from sections ext6 and ext4 have a shallow central depression as is shown in the right column of Fig. 9 where the profiles from section ext6 fitted with synthetic profiles are plotted. Plots of distributions of plasma properties for this section are shown in the right column of Fig. 10. From these plots it follows that there are rather extended PCTRs in this section (both PCTRs together occupy almost $90 \%$ of the geometrical thickness of the EUV extension in this section). In section ext4 the EUV extension is almost isothermal therefore the other plasma properties change only slightly across the 1D slab. Their values in this section are close to the values in the PCTRs at section ext6. The profiles of the hydrogen Lyman lines from sections ext7 and ext8 are similar to profiles from optically thick sections (ext1, ext2, ext5). Also values of the plasma properties are close to the values of optically thick sections but there are extended PCTRs similar to the optically thin section ext6. The sections ext7 and ext8 are optically thin at the hydrogen Lyman-continuum edge but the optical thickness $\tau_{912}$ in these sections is an order of magnitude larger than in the sections ext4 and ext6.

All the sections ext1-ext8 were chosen according to the spectra of the hydrogen Lyman lines (Fig. 3). But comparing the intensity distributions of the OVI and OV lines (Fig. 4) the hydrogen Lyman profiles from two additional parts of the SUMER slit were studied as well. One is between the sections ext5 and ext6 where the intensity of the line O VI seems to be increased very much while there is only small peak in the intensity of the OV line. It would be expected that the optical thickness resulting from the modelling will be comparable to that of ext3. But we obtained results similar to section ext5 (optical thickness $\tau_{912}$ around 2). The second additional part is a section between sections ext6 and ext7 where the intensity of the $\mathrm{OV}$ line is rather strongly decreased. Fitting the hydrogen Lyman profiles from this additional section led to similar results as were estimated for section ext6. So the EUV extension is optically thin at $912 \AA$ here also. It is in contradiction with observations of the $\mathrm{O} v$ line since the rather deep depression of intensity is observed in this additional section.

\section{Discussion and conclusions}

We have performed a detailed spectroscopic study of the EUV filament, using observations of the hydrogen Lyman lines and the results of Paper I. To analyze the conditions inside the EUV filament, we used the standard non-LTE modelling technique. However, our approach has two novel aspects. First, we have made a rather sophisticated reconstruction of the Lyman-line profiles of the background irradiation because socalled "quiet-Sun" profiles taken outside the EUV filament have 

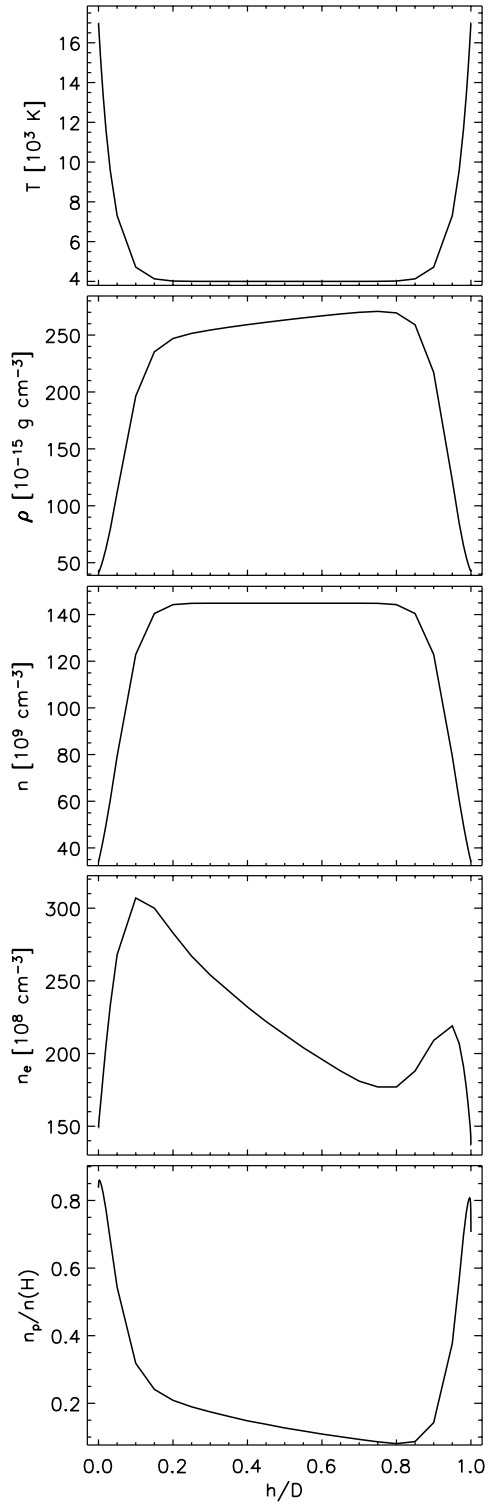
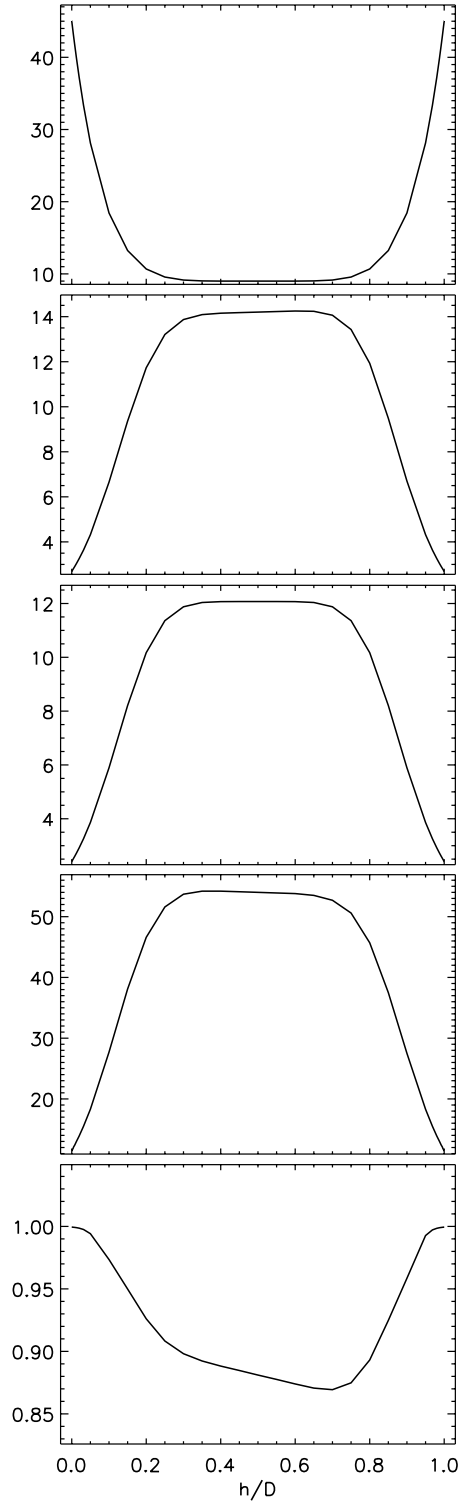
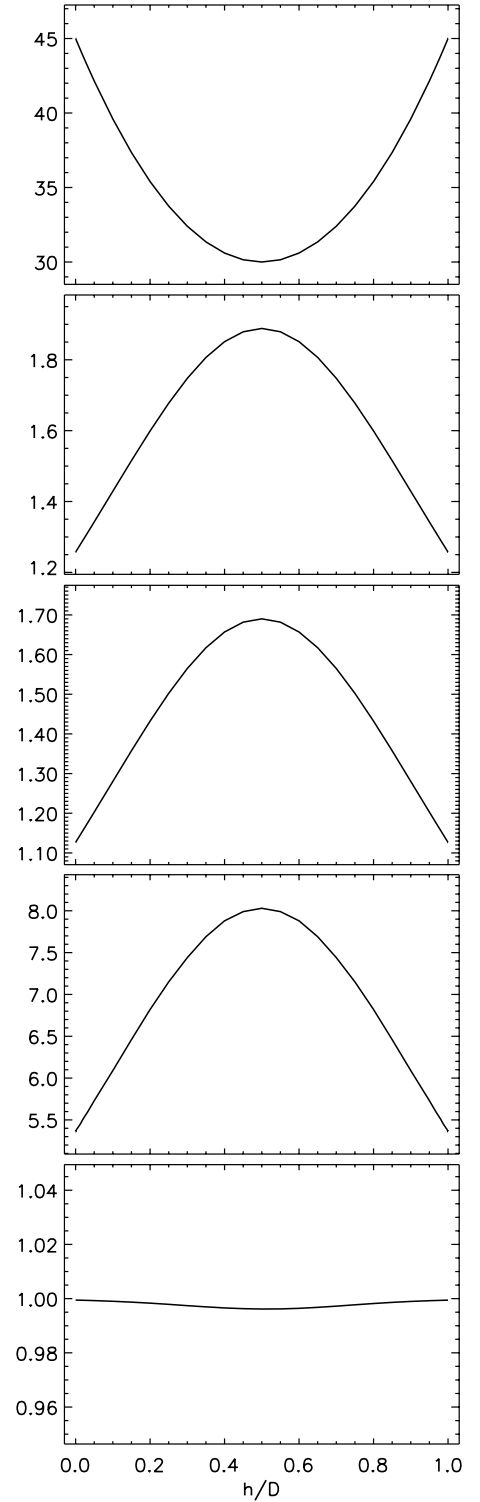

Fig. 10. Plots of distributions of plasma properties across the 1D slab: (rows from top to bottom) temperature expressed in units $10^{3} \mathrm{~K}$, plasma density in units $10^{-15} \mathrm{~g} \mathrm{~cm}^{-3}$, particle density in units $10^{9} \mathrm{~cm}^{-3}$, density of free electrons in units $10^{8} \mathrm{~cm}^{-3}$ and ionization degree of hydrogen for sections $\mathrm{H} \alpha$-fil, ext2 and ext6 (columns from left to right). Height in the filament is expressed in fractions $h / D$ of geometrical thickness $D$ of the $1 \mathrm{D}$ slab and it is measured from the bottom to top of the 1D slab.

shown higher intensities in their wings compared to profiles taken within the EUV filament. Also in the plot of the O VI line intensity (Fig. 4) the depression of this TR line within the EUV filament is clearly visible although this line is located above the Lyman-continuum edge (912 ̊) and therefore not absorbed by the hydrogen plasma of the EUV filament. Assuming that the EUV filament is placed above TR $\left(h_{\mathrm{b}}>4000 \mathrm{~km}\right)$, this intensity depression cannot be caused by the volume blocking mechanism. Therefore the depression of intensities within the EUV filament in the optically-thin wings of the hydrogen Lyman lines as well as of intensities of the O VI line can be explained only as a property of the chromosphere and TR under the filament. The reconstructed background profiles were then used to compute the synthetic profiles emerging from the EUV filament. The second novel aspect was the fitting of these synthetic profiles to observed ones using a method of extended model grids where we have searched for the best-fit model by means of the least-square optimization.
The $\mathrm{H} \alpha$ filament is geometrically thin (between several thousand and $15000 \mathrm{~km}$ ). This is the total geometrical thickness of the cool filament plasma only in the slab but the geometrical thickness of the whole slab (including cavities) can be larger. The temperature ranges from around $5000 \mathrm{~K}$ in the filament interior to $\sim 20000 \mathrm{~K}$ in the geometrically thin PCTRs. The gas

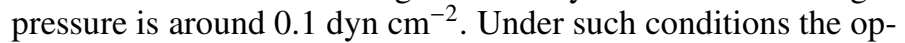
tical thickness $\tau_{\mathrm{o}}(\mathrm{H} \alpha)$ is above 1 which agrees with observations of the $\mathrm{H} \alpha$ filament in the $\mathrm{H} \alpha$ line as a very dark structure clearly seen in the chromosphere. The values of $\tau_{912}$ are of the order of magnitude $10^{2}$ and the $\mathrm{H} \alpha$ filament is geometrically thin, the absorption thus should be a more important mechanism for the intensity depression of the coronal EUV lines with wavelengths below $912 \AA$ than the volume blocking. Contrary to the $\mathrm{H} \alpha$ filament where the Lyman lines typically exhibit rather deep reversals, the Lyman profiles detected within the extensions have much shallower reversals. The coronal heights at which the EUV extension is located were derived in Paper I, assuming 
that the optical thickness $\tau_{912}$ is larger than one. Such an optical thickness was needed to explain the appearance of dark patterns in the OV line, while O VI was not affected by the absorption (compare plots of intensities of these two lines in the area between two quiet-Sun regions in Fig. 4). But the EUV extension is rather inhomogeneous; there are areas optically thick and thin in the hydrogen Lyman continuum close to each other. The temperatures in the PCTRs are 35000-50000 K and in the interior $10000-30000 \mathrm{~K}$. The gas pressure ranges from 0.01

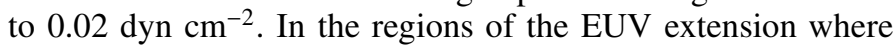
$\tau_{912}>1$ the values of $\tau_{\mathrm{o}}(\mathrm{H} \alpha)$ are below 1 . But they are larger than 0.1 at the same time, therefore it is possible to observe those regions in the $\mathrm{H} \alpha$ line as well as faint dark structures in the vicinity of the very dark and well pronounced $\mathrm{H} \alpha$ filament. Many such structures can be seen in the $\mathrm{H} \alpha$ observations of the MSDP/VTT shown in Fig. 2. The SUMER slit crosses many of them even assuming the large inaccuracy of estimation of its position in MSDP observations. Although these parts are optically thick in the hydrogen Lyman continuum, the contribution of absorption to the intensity depression is comparable to, or smaller than the contribution of the volume blocking. Thus the volume blocking is an important mechanism for the intensity depression throughout whole EUV extension and it even dominates over the absorption in many parts of the extension. In the whole EUV extension the gas pressure is very low - comparable to coronal values. In most parts of the EUV extension the filling factor is rather large (larger than 0.5) (see Table 1). Such a low pressure and large filling factor lead to a low plasma density, comparable to that of the corona. Therefore this particular EUV extension can be considered as a coronal void. The whole EUV filament is located above the TR so the volume blocking cannot influence TR lines. Then the intensity of the O V TR line is influenced only by absorption. As said above, the contribution of absorption to intensity depression is small, so only a small depression of the intensity of the OV line should be observed in the EUV extension. But the deep global intensity depression across the whole EUV filament of both oxygen lines (even of the O VI line not influenced by absorption) (see Fig. 4) and also of intensities in the optically thin wings of hydrogen Lyman lines must be the property of the TR and chromosphere themselves beneath the EUV filament.

Some questions arise from this work. Sections ext7 and ext8 belong to the EUV filament according to the depression of the $\mathrm{O}$ v line intensity (Fig. 4). But they are outside the dark structure of the CDS raster of the Mg X line (Fig. 1). This discrepancy can be explained by a tiny and faint thread belonging to the dark EUV filament which is not clearly visible in the raster. But according to the distributions along the SUMER slit of intensities of the coronal or TR lines with wavelengths below $912 \AA$, the width of the EUV filament is evident according to the intensity depression as it is shown in Fig. 4. Also depressed intensities of both the O VI TR line (not affected by absorption) and the hydrogen Lyman lines at the optically thin wings reveal this width of the EUV filament. Another question is that there are areas along the SUMER slit (e.g. ext4, ext6, section between ext6 and ext7) where the depression of the $\mathrm{O} v$ line intensity occurs but the EUV filament is optically thin at $912 \AA$. The intensity of the $\mathrm{O}$ VI line is also depressed in these sections. Therefore we can assume that the depressions of intensities of both oxygen TR lines are caused by the TR itself. On the other hand, there are also areas (ext1, section between ext5 and ext6 and especially $\mathrm{H} \alpha$-fil) where the EUV filament is optically thick at the hydrogen Lyman continuum; then a deeper depression of the $\mathrm{O} v$ line intensity would be expected. But the intensity of the O v line is not depressed or is even increased (see Fig. 4). This can be explained by the fact that the $\mathrm{O} v$ line can be emitted from hotter parts of the PCTRs. The OV and O VI TR lines are emitted at temperatures around $300000-400000 \mathrm{~K}$ (computed using the CHIANTI atomic database (Dere et al. 1997; Landi et al. 2006)). Such temperatures could not be estimated from the hydrogen spectral lines because hydrogen is totally ionized at these temperatures. Using the Lyman lines, we investigate the cooler parts of the PCTRs only where hydrogen is not totally ionized. We plan to perform study of the hotter parts of the PCTRs using the TR lines in a future paper.

Acknowledgements. This work was partly supported by grants A3003203 and 1QS300120506 of the Grant Agency of the Academy of Sciences of the Czech Republic, institutional project AV0Z10030501 and by ESA-PECS project No. 98030. SoHO/CDS and SoHO/SUMER observations were obtained during a coordinated MEDOC, THEMIS and VTT campaign. P.H., B.S. and P.S. are members of the European Solar Magnetism Network (ESMN-HPRN-CT-2002-00313) from which this work was partially supported. The SUMER data have been reduced with the intensity calibration procedure developed at MP Lindau and the wavelength calibration procedure of M. Carlsson. SoHO is a space mission of international cooperation between ESA and NASA. CHIANTI is a collaborative project involving the NRL (USA), RAL (UK), and the following Universities: College London (UK), Cambridge (UK), George Mason (USA), and Florence (Italy).

\section{References}

Anzer, U., \& Heinzel, P. 2003, A\&A, 404, 1139

Anzer, U., \& Heinzel, P. 2005, ApJ, 622, 714

Dere, K. P., Landi, E., Mason, H. E., et al. 1997, A\&AS, 125, 149

Fludra, A., del Zanna, G., Alexander, D., \& Bromage, B. J. I. 1999, J. Geophys. Res., 104, No. A5, 9709

Harrison, R. A., Sawyer, E. C., Carter, M. K., et al. 1995, Sol. Phys., 162, 233

Heinzel, P. 1995, A\&A, 299, 563

Heinzel, P., Schmieder, B., \& Tziotziou, K. 2001, ApJ, 561, L223

Heinzel, P., Schmieder, B., \& Vial, J.-C. 1997, NLTE analysis of SUMER filament observations on SoHO, in Proc. of 5th SOHO workshop, ed. A. Wilson, ESA SP-404, 427

Heinzel, P., Anzer, U., \& Schmieder, B. 2003, Sol. Phys., 216, 159

Landi, E., Del Zanna, G., Young, P. R., et al. 2006, ApJS, 162, 261

Mein, P. 1991, A\&A, 248, 669

Mein, P. 2002, A\&A, 381, 271

Paletou, F. 1995, A\&A, 302, 587

Rybicki, G. B., \& Hummer, D. G. 1991, A\&A, 245, 171

Schmieder, B., Tziotziou, K., \& Heinzel, P. 2003, A\&A, 401, 361

Schmieder, B., Lin, Y., Heinzel, P., \& Schwartz, P. 2004, Sol. Phys., 221, 297

Schwartz, P., Heinzel, P., Anzer, U., \& Schmieder, B. 2004, A\&A, 421, 323 (Paper I)

Warren, H. P., Mariska, J. T., \& Wilhelm, K. 1998, ApJS, 119, 105

Wilhelm, K., Curdt, W., Marsch, E., et al. 1995, Sol. Phys., 162, 189 